\title{
ZERO-DIMENSIONAL SYMPLECTIC ISOLATED COMPLETE INTERSECTION SINGULARITIES
}

\author{
WOJCIECH DOMITRZ
}

\begin{abstract}
We study the local symplectic algebra of the 0-dimensional isolated complete intersection singularities. We use the method of algebraic restrictions to classify these symplectic singularities. We show that there are non-trivial symplectic invariants in this classification.
\end{abstract}

\section{INTRODUCTION}

The problem of symplectic classification of singular varieties was introduced by V. I. Arnold in A1. Arnold showed that the $A_{2 k}$ singularity of a planar curve (the orbit with respect to the standard $\mathcal{A}$-equivalence of parameterized curves) split into exactly $2 k+1$ symplectic singularities (orbits with respect to the symplectic equivalence of parameterized curves). He distinguished different symplectic singularities by different orders of tangency of the parameterized curve with the nearest smooth Lagrangian submanifold. Arnold posed a problem of expressing these new symplectic invariants in terms of the local algebra's interaction with the symplectic structure and he proposed to call this interaction the local symplectic algebra. This problem was studied by many authors mainly in the case of singular curves.

In [IJ1] G. Ishikawa and S. Janeczko classified symplectic singularities of curves in the 2-dimensional symplectic space. A symplectic form on a 2-dimensional manifold is a special case of a volume form on a smooth manifold. The generalization of results in IJ1] to volume-preserving classification of singular varieties and maps in arbitrary dimensions was obtained in DR. The orbit of the action of all diffeomorphism-germs agrees with the volume-preserving orbit in the $\mathbb{C}$-analytic category for germs which satisfy a special weak form of quasi-homogeneity e.g. the weak quasi-homogeneity of varieties is a quasi-homogeneity with non-negative weights $\lambda_{i} \geq 0$ and $\sum_{i} \lambda_{i}>0$.

P. A. Kolgushkin classified stably simple symplectic singularities of parameterized curves in the $\mathbb{C}$-analytic category $([\underline{\mathrm{K}})$.

In [DJZ2 the local symplectic algebra of singular quasi-homogeneous subsets of a symplectic space was explained by the algebraic restrictions of the symplectic form to these subsets. The generalization of the Darboux-Givental theorem ( $\mathrm{AG}]$ ) to germs of arbitrary subsets of the symplectic space obtained in [DJZ2] reduces the problem of symplectic classification of germs of quasi-homogeneous subsets to the

1991 Mathematics Subject Classification. Primary 53D05. Secondary 58K40, 58K50, 58A10, $14 \mathrm{H} 20$.

Key words and phrases. symplectic manifold, local symplectic algebra, algebraic restrictions, relative Darboux theorem, isolated complete intersection singularities.

The work of W. Domitrz was supported by Polish MNiSW grant no. N N201 397237. 
problem of classification of algebraic restrictions of symplectic forms to these subsets. For non-quasi-homogeneous subsets there is one more cohomological invariant apart of the algebraic restriction (DJZ2, [DJZ1]). The method of algebraic restrictions is a very powerful tool to study the local symplectic algebra of 1-dimensional singular analytic varieties since the space of algebraic restrictions of closed 2-forms to a 1-dimensional singular analytic variety is finite-dimensional (D). By this method complete symplectic classifications of the $A-D-E$ singularities of planar curves and the $S_{5}$ singularity were obtained in DJZ2. These results were generalized to other 1-dimensional isolated complete intersection singularities: the $S_{\mu}$ symplectic singularities for $\mu>5$ in [DT1, the $T_{7}-T_{8}$ symplectic singularities in DT2] and the $W_{8}-W_{9}$ symplectic singularities in [T].

In this paper we show that some non-trivial symplectic invariants appear not only in the case of singular curves but also in the case of multiple points. We consider the symplectic classification of the 0-dimensional isolated complete intersection singularities (ICISs) in the symplectic space $\left(\mathbb{C}^{2 n}, \omega\right)$. We need to introduce a symplectic $V$-equivalence to study this problem since we consider the ideals of function-germs that have not got the property of zeros.

We recall that $\omega$ is a $\mathbb{C}$-analytic symplectic form on $\mathbb{C}^{2 n}$ if $\omega$ is a $\mathbb{C}$-analytic nondegenerate closed 2-form, and $\Phi: \mathbb{C}^{2 n} \rightarrow \mathbb{C}^{2 n}$ is a symplectomorphism if $\Phi$ is a $\mathbb{C}$-analytic diffeomorphism and $\Phi^{*} \omega=\omega$.

Definition 1.1. Let $f, g:\left(\mathbb{C}^{2 n}, 0\right) \rightarrow\left(\mathbb{C}^{k}, 0\right)$ be $\mathbb{C}$-analytic map-germs on the symplectic space $\left(\mathbb{C}^{2 n}, \omega\right) . f, g$ are symplectically $V$-equivalent if there exist a symplectomorphism-germ $\Phi:\left(\mathbb{C}^{2 n}, 0, \omega\right) \rightarrow\left(\mathbb{C}^{2 n}, 0, \omega\right)$ and a $\mathbb{C}$-analytic map-germ $M:\left(\mathbb{C}^{2 n}, 0\right) \rightarrow G L(k, \mathbb{C})$ such that such that $f \circ \Phi=M \cdot g$.

If $\Phi:\left(\mathbb{C}^{n}, 0\right) \rightarrow\left(\mathbb{C}^{m}, 0\right)$ is a $\mathbb{C}$-analytic map-germ then for an ideal $I$ in the ring of $\mathbb{C}$-analytic function-germs on $\mathbb{C}^{m}$ we denote by $\Phi^{*} I$ the following ideal $\{f \circ \Phi: f \in I\}$ in the ring of $\mathbb{C}$-analytic function-germs on $\mathbb{C}^{n}$. The (symplectic) $V$-equivalence of map-germs $f=\left(f_{1}, \cdots, f_{k}\right), g=\left(g_{1}, \cdots, g_{k}\right):\left(\mathbb{C}^{2 n}, 0\right) \rightarrow\left(\mathbb{C}^{k}, 0\right)$ corresponds to the following (symplectic) equivalence of finitely-generated ideals $<f_{1}, \cdots, f_{k}>$ and $<g_{1}, \cdots, g_{k}>$ (see AVG]).

Definition 1.2. Ideals $\left\langle f_{1}, \cdots, f_{k}>\right.$ and $\left\langle g_{1}, \cdots, g_{k}>\right.$ of $\mathbb{C}$-analytic functiongerms at 0 on the symplectic space $\left(\mathbb{C}^{2 n}, \omega\right)$ are symplectically equivalent if there exists a symplectomorphism-germ $\Phi:\left(\mathbb{C}^{2 n}, 0, \omega\right) \rightarrow\left(\mathbb{C}^{2 n}, 0, \omega\right)$ such that $\Phi^{*}<f_{1}, \cdots, f_{k}>=<g_{1}, \cdots, g_{k}>$.

In this paper we present the complete symplectic classification of the $I_{a, b}, I_{2 a+1}$, $I_{2 a+4}, I_{a+5}, I_{10}^{*}$ singularities. For $n=1$ all $V$-orbits coincide with symplectic $V$ orbits. The situation for $n \geq 2$ is different: the $I_{a, b}$ singularities split into two symplectic $V$-orbits, the $I_{2 a+1}, I_{2 a+4}, I_{a+5}$ singularities split into three symplectic orbits and finally $I_{10}^{*}$ singularity splits into four symplectic $V$-orbits. The symplectic $V$-orbits of a map $f=\left(f_{1}, \cdots, f_{2 n}\right)$ are distinguished by the order of vanishing of a pullback of the germ of the symplectic form to a $\mathbb{C}$-analytic non-singular submanifold $M$ of the minimal dimension such that the ideal of $\mathbb{C}$-analytic function-germs vanishing $M$ is contained in the ideal $\left\langle f_{1}, \cdots, f_{2 n}\right\rangle$ (see Definition 3.2).

To obtain these results we need some reformulation and modification of the method of algebraic restrictions. We present it in Section 2, In Section 3 we give the definitions of discrete symplectic invariants which completely distinguish symplectic $V$-singularities considered in this paper. We recall basic facts on the 
classification of $V$-simple maps in Section 4 In Section 5 we prove the symplectic $V$-classification theorem for 0-dimensional ICISs (Theorem 5.1).

\section{The Method of Algebraic Restrictions For the SYMPleCtiC $V$-EQUIVALENCE.}

In this section we present basic facts on the method of algebraic restrictions adapted to the case of the symplectic $V$-equivalence. The proofs of all results are small modifications of the proofs of analogous results in DJZ2.

Given a germ at 0 of a non-singular $\mathbb{C}$-analytic submanifold $M$ of $\mathbb{C}^{m}$ denote by $\Lambda^{p}(M)$ the space of all germs at 0 of $\mathbb{C}$-analytic differential $p$-forms on $M$. By $\mathcal{O}(M)$ denote the ring of $\mathbb{C}$-analytic function-germs on $M$ at 0 . Given an ideal $I$ in $\mathcal{O}(M)$ introduce the following subspace of $\Lambda^{p}(M)$ :

$$
\mathcal{A}_{0}^{p}(I, M)=\left\{\alpha+d \beta: \quad \alpha \in I \Lambda^{p}(M), \beta \in I \Lambda^{p-1}(M) .\right\}
$$

The relation $\omega \in I \Lambda^{p}(M)$ means that $\omega=\sum_{i=1}^{k} f_{i} \alpha_{i}$, where $\alpha_{i} \in \Lambda^{p}(M)$ and $f_{i} \in I$ for $i=1, \ldots, k$.

Definition 2.1. Let $I$ be an ideal of $\mathcal{O}(M)$ and let $\omega \in \Lambda^{p}(M)$. The algebraic restriction of $\omega$ to $I$ is the equivalence class of $\omega$ in $\Lambda^{p}(M)$, where the equivalence is as follows: $\omega$ is equivalent to $\widetilde{\omega}$ if $\omega-\widetilde{\omega} \in \mathcal{A}_{0}^{p}(I, M)$.

Notation. The algebraic restriction of the germ of a $p$-form $\omega$ on $M$ to the ideal $I$ in $\mathcal{O}(M)$ will be denoted by $[\omega]_{I}$. Writing $[\omega]_{I}=0$ (or saying that $\omega$ has zero algebraic restriction to $I$ ) we mean that $[\omega]_{I}=[0]_{I}$, i.e. $\omega \in A_{0}^{p}(I, M)$.

Definition 2.2. Two algebraic restrictions $[\omega]_{I}$ and $[\widetilde{\omega}]_{\tilde{I}}$ are called diffeomorphic if there exists the germ of a diffeomorphism $\Phi: M \rightarrow \widetilde{M}$ such that $\Phi^{*}(\widetilde{I})=I$ and $\left[\Phi^{*} \widetilde{\omega}\right]_{I}=[\omega]_{I}$.

Definition 2.3. The germ of a function, a differential $k$-form, or a vector field $\alpha$ on $\left(\mathbb{C}^{m}, 0\right)$ is quasi-homogeneous in a coordinate system $\left(x_{1}, \cdots, x_{m}\right)$ on $\left(\mathbb{C}^{m}, 0\right)$ with positive integer weights $\left(\lambda_{1}, \cdots, \lambda_{m}\right)$ if $\mathcal{L}_{E} \alpha=\delta \alpha$, where $E=\sum_{i=1}^{m} \lambda_{i} x_{i} \frac{\partial}{\partial x_{i}}$ is the germ of the Euler vector field on $\left(\mathbb{C}^{m}, 0\right)$ and the integer $\delta$ is called the quasi-degree.

It is easy to show that $\alpha$ is quasi-homogeneous in a coordinate system $\left(x_{1}, \cdots, x_{m}\right)$ with weights $\left(\lambda_{1}, \cdots, \lambda_{m}\right)$ if and only if $F_{t}^{*} \alpha=t^{\delta} \alpha$, where

$$
F_{t}\left(x_{1}, \cdots, x_{m}\right)=\left(t^{\lambda_{1}} x_{1}, \cdots, t^{\lambda_{m}} x_{m}\right) .
$$

Definition 2.4. A finitely generated ideal $I$ of $\mathcal{O}\left(\mathbb{C}^{m}\right)$ is quasi-homogeneous if there exist generators of $I$ which are quasi-homogeneous in the same coordinate system $\left(x_{1}, \cdots, x_{m}\right)$ on $\mathbb{C}^{m}$ with the same positive integer weights $\left(\lambda_{1}, \cdots, \lambda_{m}\right)$.

A map-germ $f=\left(f_{1}, \cdots, f_{k}\right):\left(\mathbb{C}^{m}, 0\right) \rightarrow\left(\mathbb{C}^{k}, 0\right)$ is quasi-homogeneous if function-germs $f_{1}, \cdots, f_{k}$ are quasi-homogeneous in the same coordinate system $\left(x_{1}, \cdots, x_{m}\right)$ on $\mathbb{C}^{m}$ with the same positive integer weights $\left(\lambda_{1}, \cdots, \lambda_{m}\right)$.

To prove the generalization of Darboux-Givental theorem suitable for the symplectic $V$-equivalence of maps or the symplectic equivalence of ideals of functiongerms we need the following version of the Relative Poincaré Lemma.

Lemma 2.5. Let I be a finitely generated quasi-homogeneous ideal in $\mathcal{O}\left(\mathbb{C}^{m}\right)$. If $\omega \in I \Lambda^{p}\left(\mathbb{C}^{m}\right)$ is closed than there exists $\alpha \in I \Lambda^{p-1}\left(\mathbb{C}^{m}\right)$ such that $\omega=d \alpha$. 
Proof. We use the method described in DJZ1. We can find a coordinate system $\left(x_{1}, \cdots, x_{m}\right)$ on $\left(\mathbb{C}^{m}, 0\right)$ and positive integer weights $\left(\lambda_{1}, \cdots, \lambda_{m}\right)$ and quasihomogeneous function-germs $f_{1}, \cdots, f_{k} \in \mathcal{O}\left(\mathbb{C}^{m}\right)$ (in this coordinate systems with these weights) such that $I=<f_{1}, \cdots, f_{k}>$. Let $\delta_{i}$ be a quasi-degree of $f_{i}$ for $i=1, \cdots, k$.

Let $F_{t}$ be a map defined in (2.1) and let $V_{t}$ be a vector field along $F_{t}$ for $t \in[0 ; 1]$ such that $V_{t} \circ F_{t}=F_{t}^{\prime}$.

Then we have $F_{0}^{*} \omega=0$ and it implies that

$$
\left.\left.\omega=F_{1}^{*} \omega-F_{0}^{*} \omega=\int_{0}^{1}\left(F_{t}^{*} \omega\right)^{\prime} d t=\int_{0}^{1} F_{t}^{*} d\left(V_{t}\right\rfloor \omega\right) d t=d\left(\int_{0}^{1} F_{t}^{*}\left(V_{t}\right\rfloor \omega\right) d t\right) .
$$

Let $\left.\alpha=\int_{0}^{1} F_{t}^{*}\left(V_{t}\right\rfloor \omega\right) d t$, then $\omega=d \alpha$. But $\omega$ belongs to $I \Lambda^{p}\left(\mathbb{C}^{m}\right)$. It implies that there exist germs of $p$-forms $\beta_{i}$ in $\Lambda^{p}\left(\mathbb{C}^{m}\right)$ for $i=1, \cdots, k$ such that $\omega=\sum_{i=1}^{k} f_{i} \beta_{i}$. So we have that

$$
\left.\left.\alpha=\int_{0}^{1} F_{t}^{*}\left(V_{t}\right\rfloor \sum_{i=1}^{k} f_{i} \beta_{i}\right) d t=\sum_{i=1}^{k} f_{i} \int_{0}^{1} t^{\delta_{i}} F_{t}^{*}\left(V_{t}\right\rfloor \beta_{i}\right) d t .
$$

Thus $\alpha$ belongs to $I \Lambda^{p-1}\left(\mathbb{C}^{m}\right)$.

The method of algebraic restrictions applied to finitely-generated quasi-homogeneous ideals is based on the following theorem.

Theorem 2.6 (a modification of Theorem A in DJZ2]). Let I be a finitely generated quasi-homogeneous ideal in $\mathcal{O}\left(\mathbb{C}^{2 n}\right)$.

(1) If $\omega_{0}, \omega_{1}$ are germs at 0 of symplectic forms on $\mathbb{C}^{2 n}$ with the same algebraic restriction to $I$ then there exists a $\mathbb{C}$-analytic diffeomorphism-germ $\Phi$ of $\mathbb{C}^{2 n}$ at 0 of the form $\Phi(x)=\left(x_{1}+\phi_{1}(x), \cdots, x_{2 n}+\phi_{2 n}(x)\right)$, where $\phi_{i} \in I$ for $i=1, \cdots, 2 n$, such that $\Phi^{*} \omega_{1}=\omega_{0}$.

(2) $\mathbb{C}$-analytic quasi-homogeneous map-germs $f=\left(f_{1}, \cdots, f_{k}\right), g=\left(g_{1}, \cdots, g_{k}\right)$ : $\left(\mathbb{C}^{2 n}, 0\right) \rightarrow\left(\mathbb{C}^{k}, 0\right)$ on the symplectic space $\left(\mathbb{C}^{2 n}, \omega\right)$ are symplectically $V$ equivalent if and only if algebraic restrictions $[\omega]_{\left.<f_{1}, \cdots, f_{k}\right\rangle}$ and $[\omega]_{\left\langle g_{1}, \cdots, g_{k}\right\rangle}$ are diffeomorphic.

Remark 2.7. It is obvious that if $\Phi(x)=\left(x_{1}+\phi_{1}(x), \cdots, x_{2 n}+\phi_{2 n}(x)\right)$ where $\phi_{i} \in I$ for $i=1, \cdots, 2 n$ then $\Phi^{*} I=I$

A proof of Theorem 2.6 can be obtain by a small modification of the proof of Theorem A in DJZ2. One only needs Lemma 2.5 and the following fact.

Lemma 2.8. Let $I$ be a finitely generated ideal in $\mathcal{O}\left(\mathbb{C}^{m}\right)$. Let $X_{t}=\sum_{i=1}^{m} f_{i, t} \frac{\partial}{\partial x_{i}}$ for $t \in[0 ; 1]$ be a family of germs of $\mathbb{C}$-analytic vector fields on $\mathbb{C}^{m}$ such that $f_{i, t} \in I$ for $i=1, \cdots, m$.

If $\Phi_{t}$ for $t \in[0,1]$ is a family of diffeomorphism-germs of $\left(\mathbb{C}^{m}, 0\right)$ such that

$$
\frac{d}{d t} \Phi_{t}=X_{t} \circ \Phi_{t}
$$

then

$$
\Phi_{t}(x)=\left(x_{1}+\phi_{1, t}(x), \cdots, x_{2 n}+\phi_{2 n, t}(x)\right),
$$

where $\phi_{i, t} \in I$ for $i=1, \cdots, 2 n$. 
$A$ sketch of the proof. The map $t \mapsto \Phi_{t}(x)$ is a solution of ODE $\frac{d y}{d t}=X_{t}(y)$ with the initial condition $y(0)=x$. So $\Phi_{t}(x)$ can be obtained as a $\operatorname{limit}_{n \rightarrow \infty} T^{n} \Psi$ where $\Psi(t, x) \equiv x$ and $(T \Psi)(t, x)=x+\int_{0}^{t} X_{s}(\Psi(s, x)) d s$ is the Picard's operator. It is easy to see that if $\Psi$ has the form (2.3) then $T \Psi$ has the form (2.3) too. The ideal $I$ is finitely generated. Thus $\Phi_{t}$ has also this form.

Theorem 2.6 reduces the problem of symplectic classification of quasi-homogeneous ideals to the problem of classification of the algebraic restrictions of the germ of the symplectic form to quasi-homogeneous ideals.

The meaning of the zero algebraic restriction is explained by the following theorem.

Theorem 2.9 (a modification of Theorem B in [DJZ2]). A finitely generated quasihomogeneous ideal $I$ of $\mathcal{O}\left(\mathbb{C}^{2 n}\right)$ contains the ideal of $\mathbb{C}$-analytic function-germs vanishing on the germ of a non-singular Lagrangian submanifold of the symplectic space $\left(\mathbb{C}^{2 n}, \omega\right)$ if and only if the symplectic form $\omega$ has zero algebraic restriction to $I$.

We now formulate the modifications of basic properties of algebraic restrictions (DJZ2 ]). First we can reduce the dimension of the manifold due to the following propositions.

If the ideal $I$ in $\mathcal{O}\left(\mathbb{C}^{m}\right)$ contains an ideal $I(M)$ of function-germs vanishing on a non-singular submanifold $M \subset \mathbb{C}^{m}$ then the classification of the algebraic restrictions to $I$ of $p$-forms on $\mathbb{C}^{m}$ reduces to the classification of the algebraic restrictions to $\left.I\right|_{M}=\left\{\left.f\right|_{M}: f \in I\right\}$ of $p$-forms on $M$. At first note that the algebraic restrictions $[\omega]_{I}$ and $\left[\left.\omega\right|_{T M}\right]_{\left.I\right|_{M}}$ can be identified:

Proposition 2.10. Let I be an ideal in $\mathcal{O}\left(\mathbb{C}^{m}\right)$ which contains an ideal of functiongerms vanishing on a non-singular submanifold $M \subset \mathbb{C}^{m}$ and let $\omega_{1}, \omega_{2}$ be germs of $p$-forms on $\mathbb{C}^{m}$. Then $\left[\omega_{1}\right]_{I}=\left[\omega_{2}\right]_{I}$ if and only if $\left[\left.\omega_{1}\right|_{T M}\right]_{\left.I\right|_{M}}=\left[\left.\omega_{2}\right|_{T M}\right]_{\left.I\right|_{M}}$.

The following, less obvious statement, means that the orbits of the algebraic restrictions $[\omega]_{I}$ and $\left[\left.\omega\right|_{T M}\right]_{\left.I\right|_{M}}$ also can be identified.

Proposition 2.11. Let $I_{1}, I_{2}$ be ideals in the ring $\mathcal{O}\left(\mathbb{C}^{m}\right)$, which contain $I\left(M_{1}\right)$ and $I\left(M_{2}\right)$ respectively, where $M_{1}, M_{2}$ are equal-dimensional non-singular submanifolds. Let $\omega_{1}, \omega_{2}$ be two germs of p-forms. The algebraic restrictions $\left[\omega_{1}\right]_{I_{1}}$ and $\left[\omega_{2}\right]_{I_{2}}$ are diffeomorphic if and only if the algebraic restrictions $\left[\left.\omega_{1}\right|_{T M_{1}}\right]_{\left.I_{1}\right|_{M_{1}}}$ and $\left[\left.\omega_{2}\right|_{T M_{2}}\right]_{\left.I_{2}\right|_{M_{2}}}$ are diffeomorphic.

To calculate the space of algebraic restrictions of germs of 2 -forms we will use the following obvious properties.

Proposition 2.12. If $\omega \in \mathcal{A}_{0}^{k}\left(I, \mathbb{C}^{2 n}\right)$ then $d \omega \in \mathcal{A}_{0}^{k+1}\left(I, \mathbb{C}^{2 n}\right)$ and $\omega \wedge \alpha \in$ $\mathcal{A}_{0}^{k+p}\left(I, \mathbb{C}^{2 n}\right)$ for any germ of $\mathbb{C}$-analytic $p$-form $\alpha$ on $\mathbb{C}^{2 n}$.

Then we need to determine which algebraic restrictions of closed 2-forms are realizable by symplectic forms. This is possible due to the following fact.

Proposition 2.13. Let $I$ be an ideal of $\mathcal{O}\left(\mathbb{C}^{2 n}\right)$. Let $r$ be the minimal dimension of non-singular submanifolds $M$ of $\mathbb{C}^{2 n}$ such that I contains the ideal $I(M)$. The algebraic restriction $[\theta]_{I}$ of the germ of a closed 2 -form $\theta$ is realizable by the germ of a symplectic form on $\mathbb{C}^{2 n}$ if and only if $\operatorname{rank}\left(\left.\theta\right|_{T_{0} M}\right) \geq 2 r-2 n$. 


\section{DisCRETE SYMPLECTIC INVARIANTS.}

We use discrete symplectic invariants to distinguish symplectic singularity classes. We modify definitions of these invariants introduced in DJZ2 for the symplectic $V$-equivalence.

The first invariant is a symplectic multiplicity ([DJZ2]) introduced in [IJ1] as a symplectic defect of a curve.

Let $f:\left(\mathbb{C}^{2 n}, 0\right) \rightarrow\left(\mathbb{C}^{k}, 0\right)$ be the germ of a $\mathbb{C}$-analytic map on the symplectic space $\left(\mathbb{C}^{2 n}, \omega\right)$.

Definition 3.1. The symplectic multiplicity $\mu_{\text {sympl }}(f)$ of $f$ is the codimension of the symplectic $V$-orbit of $f$ in the $V$-orbit of $f$.

The second invariant is the index of isotropy [DJZ2].

Definition 3.2. The index of isotropy $\iota(f)$ of $f=\left(f_{1}, \cdots, f_{k}\right)$ is the maximal order of vanishing of the 2 -forms $\left.\omega\right|_{T M}$ over all smooth submanifolds $M$ such that the ideal $<f_{1}, \cdots, f_{k}>$ contains $I(M)$.

These invariants can be described in terms of algebraic restrictions.

Proposition 3.3 (DJZ2]). The symplectic multiplicity of the germ of a quasihomogeneous map $f=\left(f_{1}, \cdots, f_{k}\right)$ on the symplectic space $\left(\mathbb{C}^{2 n}, \omega\right)$ is equal to the

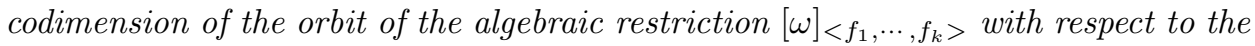
group of diffeomorphism-germs preserving the ideal $<f_{1}, \cdots, f_{k}>$ in the space of the algebraic restrictions of closed 2 -forms to $\left\langle f_{1}, \cdots, f_{k}\right\rangle$.

Proposition 3.4 ([DJZ2]). The index of isotropy of the germ of a quasi-homogeneous map $f=\left(f_{1}, \cdots, f_{k}\right)$ on the symplectic space $\left(\mathbb{C}^{2 n}, \omega\right)$ is equal to the maximal order

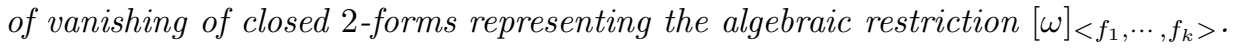

We will use these invariants to distinguish symplectic singularities.

\section{4. $V$-SIMPLE MAPS}

We recall some results on classification of $V$-simple germs (for details see [AVG]).

Definition 4.1. The germ $f:\left(\mathbb{C}^{m}, 0\right) \rightarrow\left(\mathbb{C}^{n}, 0\right)$ is said be $V$-simple if its $k$-jet, for any $k$, has a neighborhood in the small jet space $J_{0,0}^{k}\left(\mathbb{C}^{m}, \mathbb{C}^{n}\right)$ that intersects only a finite number of $V$-equivalence classes (bounded by a constant independent of $k)$.

Definition 4.2. The $p$-parameter suspension of the map-germ $f:\left(\mathbb{C}^{m}, 0\right) \rightarrow$ $\left(\mathbb{C}^{n}, 0\right)$ is the map germ

$$
F:\left(\mathbb{C}^{m} \times \mathbb{C}^{p}, 0\right) \ni(y, z) \mapsto(f(y), z) \in\left(\mathbb{C}^{n} \times \mathbb{C}^{p}, 0\right) .
$$

Theorem 4.3 (see $\mathrm{AVG}]$ ). The $V$-simple map-germs $\left(\mathbb{C}^{m}, 0\right) \rightarrow\left(\mathbb{C}^{n}, 0\right)$ with $m \geq$ $n$ belong, up to $V$-equivalence and suspension, to one of the three lists: the $A-D-E$ singularities of map-germs $\mathbb{C}^{m} \rightarrow \mathbb{C}$ (hypersurfaces with an isolated singularity), $S-T-U-W-Z$ singularities of map-germs $\mathbb{C}^{3} \rightarrow \mathbb{C}^{2}$ (1-dimensional ICISs) and singularities of map-germs $\mathbb{C}^{2} \rightarrow \mathbb{C}^{2}$ (0-dimensional ICISs) presented in Table 1.

The normal forms in Table 1 were obtained in $\mathrm{G}$ by M. Giusti. 


\begin{tabular}{|c|c|c|}
\hline Notation & Normal form & Restrictions \\
\hline$I_{a, b}$ & $\left(y z, y^{a}+z^{b}\right)$ & $a \geq b \geq 2$ \\
\hline$I_{2 a+1}$ & $\left(y^{2}+z^{3}, z^{a}\right)$ & $a \geq 3$ \\
\hline$I_{2 a+4}$ & $\left(y^{2}+z^{3}, y z^{a}\right)$ & $a \geq 2$ \\
\hline$I_{a+5}$ & $\left(y^{2}+z^{a}, y z^{2}\right)$ & $a \geq 4$ \\
\hline$I_{10}^{*}$ & $\left(y^{2}, z^{4}\right)$ & - \\
\hline
\end{tabular}

TABLE 1 . V-simple map-germs $\mathbb{C}^{2} \rightarrow \mathbb{C}^{2}$.

\section{SympleCtic 0-Dimensional ICISs}

We use the method of algebraic restrictions to obtain a complete classification of singularities presented in Table 1.

Theorem 5.1. Any map-germ $\left(\mathbb{C}^{2 n}, 0\right) \rightarrow\left(\mathbb{C}^{2 n}, 0\right)$ from the symplectic space $\left(\mathbb{C}^{2 n}, \sum_{i=1}^{n} d p_{i} \wedge d q_{i}\right)$ which is $V$-equivalent (up to a suitable suspension) to one of the normal forms in Table 1 is symplectically $V$-equivalent to one and only one of the following normal forms presented in Table 2

\begin{tabular}{|c|c|c|c|c|}
\hline Symplectic class & Normal forms & cod & $\mu_{\text {sympl }}$ & $i$ \\
\hline$I_{a, b}^{0},(n \geq 1)$ & $\left(p_{1} q_{1}, p_{1}^{a}+q_{1}^{b}, p_{2}, q_{2}, \cdots, p_{n}, q_{n}\right)$ & 0 & 0 & 0 \\
\hline$I_{a, b}^{1},(n \geq 2)$ & $\left(p_{1} p_{2}, p_{1}^{a}+p_{2}^{b}, q_{1}, q_{2}, p_{3}, q_{3}, \cdots, p_{n}, q_{n}\right)$ & 1 & 1 & $\infty$ \\
\hline \hline$I_{2 a+1}^{0},(n \geq 1)$ & $\left(p_{1}^{2}+q_{1}^{3}, q_{1}^{a}, p_{2}, q_{2}, \cdots, p_{n}, q_{n}\right)$ & 0 & 0 & 0 \\
\hline$I_{2 a+1}^{1},(n \geq 2)$ & $\left(p_{1}^{2}+p_{2}^{3}, p_{2}^{a}, q_{1}, q_{2}+p_{1} p_{2}, p_{3}, q_{3}, \cdots, p_{n}, q_{n}\right)$ & 1 & 1 & 1 \\
\hline$I_{2 a+1}^{2},(n \geq 2)$ & $\left(p_{1}^{2}+p_{2}^{3}, p_{2}^{a}, q_{1}, q_{2}, p_{3}, q_{3}, \cdots, p_{n}, q_{n}\right)$ & 2 & 2 & $\infty$ \\
\hline \hline$I_{2 a+4}^{0},(n \geq 1)$ & $\left(p_{1}^{2}+q_{1}^{3}, p_{1} q_{1}^{a}, p_{2}, q_{2}, \cdots, p_{n}, q_{n}\right)$ & 0 & 0 & 0 \\
\hline$I_{2 a+4}^{1},(n \geq 2)$ & $\left(p_{1}^{2}+p_{2}^{3}, p_{1} p_{2}^{a}, q_{1}, q_{2}+p_{1} p_{2}, p_{3}, q_{3}, \cdots, p_{n}, q_{n}\right)$ & 1 & 1 & 1 \\
\hline$I_{2 a+4}^{2},(n \geq 2)$ & $\left(p_{1}^{2}+p_{2}^{3}, p_{1} p_{2}^{a}, q_{1}, q_{2}, p_{3}, q_{3}, \cdots, p_{n}, q_{n}\right)$ & 2 & 2 & $\infty$ \\
\hline \hline$I_{a+5}^{0},(n \geq 1)$ & $\left(p_{1}^{2}+q_{1}^{a}, p_{1} q_{1}^{2}, p_{2}, q_{2}, \cdots, p_{n}, q_{n}\right)$ & 0 & 0 & 0 \\
\hline$I_{a+5}^{1},(n \geq 2)$ & $\left(p_{1}^{2}+p_{2}^{a}, p_{1} p_{2}^{2}, q_{1}, q_{2}+p_{1} p_{2}, p_{3}, q_{3}, \cdots, p_{n}, q_{n}\right)$ & 1 & 1 & 1 \\
\hline$I_{a+5}^{1},(n \geq 2)$ & $\left(p_{1}^{2}+p_{2}^{a}, p_{1} p_{2}^{2}, q_{1}, q_{2}, p_{3}, q_{3}, \cdots, p_{n}, q_{n}\right)$ & 2 & 2 & $\infty$ \\
\hline \hline$I_{10}^{* 0},(n \geq 1)$ & $\left(p_{1}^{2}, q_{1}^{4}, p_{2}, q_{2}, \cdots, p_{n}, q_{n}\right)$ & 0 & 0 & 0 \\
\hline$I_{10}^{* 1},(n \geq 2)$ & $\left(p_{1}^{2}, p_{2}^{4}, q_{1}, q_{2}+p_{1} p_{2}, p_{3}, q_{3}, \cdots, p_{n}, q_{n}\right)$ & 1 & 1 & 1 \\
\hline$I_{10}^{* 2},(n \geq 2)$ & $\left(p_{1}^{2}, p_{2}^{4}, q_{1}, q_{2}+p_{1} p_{2}^{2}, p_{3}, q_{3}, \cdots, p_{n}, q_{n}\right)$ & 2 & 2 & 2 \\
\hline$I_{10}^{* 3},(n \geq 2)$ & $\left(p_{1}^{2}, p_{2}^{4}, q_{1}, q_{2}, p_{3}, q_{3}, \cdots, p_{n}, q_{n}\right)$ & 3 & 3 & $\infty$ \\
\hline
\end{tabular}

TABLE 2. Classification of symplectic 0-dimensional isolated complete intersection singularities, cod - codimension of the classes; $\mu_{s y m p l}{ }^{-}$ symplectic multiplicity; $i$ - index of isotropy.

Proof. In the case $n=1$ the proof follows from results in [DR] where it was proved that for quasi-homogeneous singularities in the $\mathbb{C}$-analytic category $V$-orbits coincide with volume-preserving $V$-orbits. For general $n$ we present the proof in the case of the $I_{10}^{*}$ singularity where there are 4 different symplectic singularity classes, and in the case of the $I_{a+5}$ singularity. The proofs in other cases are very similar.

For the $I_{10}^{*}$ singularity we calculate the space of algebraic restrictions of 2-forms to the ideal $I=<y^{2}, z^{4}, x_{1}, \cdots, x_{2 n-2}>$. The ideal generated by $x_{1}, \cdots, x_{2 n-2}$ is contained in $I$. So by Proposition 2.10 we may consider the following ideal $J=\left.I\right|_{\left\{x_{1}=\cdots=x_{2 n-2}=0\right\}}=<y^{2}, z^{4}>$ in the ring $\mathcal{O}\left(\mathbb{C}^{2}\right)$. By Proposition 2.12 germs 
of 1-forms $d\left(1 / 2 y^{2}\right)=y d y, d\left(1 / 4 z^{4}\right)=z^{3} d z$ and germs of 2-forms $y d y \wedge d z, z^{3} d y \wedge d z$ have zero algebraic restriction to $J$. So any algebraic restriction of the germ of a closed 2-forms to $J$ can be presented in the following form $[\omega]_{J}=A[d y \wedge d z]_{J}+$ $B[z d y \wedge d z]_{J}+C\left[z^{2} d y \wedge d z\right]_{J}$, where $A, B, C \in \mathbb{C}$.

If $A \neq 0$ then we obtain $\Phi^{*}[\omega]_{J}=[d y \wedge d z]_{J}$ by the diffeomorphism-germ of the form $\Phi(y, z)=\left(y, z\left(A+1 / 2 B z+1 / 3 C z^{2}\right)\right)$. If $A=0$ and $B \neq 0$ then we obtain $\Phi^{*}[\omega]_{J}=[z d y \wedge d z]_{J}$ by the diffeomorphism-germ of the form $\Phi(y, z)=(y, z \phi(z))$, where $\phi^{2}(z)=B+2 / 3 C z$. If $A=B=0$ and $C \neq 0$ then we obtain $\Phi^{*}[\omega]_{J}=$ $\left[z^{2} d y \wedge d z\right]_{J}$ by the diffeomorphism-germ of the form $\Phi(y, z)=(C y, z)$.

Since the minimal dimension $r$ of the germ of a non-singular submanifold $M$ such that $I(M) \subset I$ is 2 then by Proposition 2.13 for $n=1$ only the algebraic restriction $[d y \wedge d z]_{I}$ is realizable by the germ of a symplectic form.

For $n>1$ all algebraic restrictions are realizable by the following symplectic forms:

$$
\begin{gathered}
d y \wedge d z+\sum_{i=1}^{n-1} d x_{2 i-1} \wedge d x_{2 i} \\
z d y \wedge d z+d y \wedge d x_{1}+d z \wedge d x_{2}+\sum_{i=2}^{n-1} d x_{2 i-1} \wedge d x_{2 i}, \\
z^{2} d y \wedge d z+d y \wedge d x_{1}+d z \wedge d x_{2}+\sum_{i=2}^{n-1} d x_{2 i-1} \wedge d x_{2 i}, \\
d y \wedge d x_{1}+d z \wedge d x_{2}+\sum_{i=2}^{n-1} d x_{2 i-1} \wedge d x_{2 i} .
\end{gathered}
$$

By a simple change of coordinates we obtain the normal forms in Table 2.

For the $I_{a+5}$ singularity the space algebraic restrictions of germs of closed 2forms to the ideal $I=<y^{2}+z^{a}, y z^{2}, x_{1}, \cdots, x_{2 n-2}>$ can calculated in the same way. We obtain that any algebraic restriction of the germs of a closed 2-forms on $\mathbb{C}^{2}=\left\{x_{1}=\cdots=x_{2 n-2}=0\right\}$ to $J=\left.I\right|_{\left\{x_{1}=\cdots=x_{2 n-2}=0\right\}}=<y^{2}+z^{a}, y z^{2}>$ can be presented in the following form

$$
[\omega]_{J}=A[d y \wedge d z]_{J}+B[z d y \wedge d z]_{J},
$$

where $A, B \in \mathbb{C}$.

First assume that $A \neq 0$. Let $E$ denote the germ of the Euler vector field $a y \frac{\partial}{\partial y}+2 z \frac{\partial}{\partial y}$. Then it is easy to check that a flow $\Phi_{t}$ of the germ of a vector field $X=\frac{B}{(a+4) A} z E$ preserves $J, \mathcal{L}_{X}(A d y \wedge d z)=B z d y \wedge d z,\left[\mathcal{L}_{X}(B z d y \wedge d z)\right]_{J}=0$. Therefore $\Phi_{t}^{*}[A d y \wedge d z+t B z d y \wedge d z]_{J}=[A d y \wedge d z]_{J}$ for $t \in[0 ; 1]$ (see [D]). Finally by a linear change of coordinates of the form $(y, z) \mapsto(C y, D z)$, where for $C, D \in \mathbb{C}$ such that $C^{2}=D^{a}$ and $C D=A$ we show that if $A \neq 0$ then the algebraic restriction (5.5) is diffeomorphic to $[d y \wedge d z]_{J}$. By a similar change of coordinates preserving $J$ we show that if $A=0$ and $B \neq 0$ then the algebraic restriction (5.5) is diffeomorphic to $[z d y \wedge d z]_{J}$. As in the previous case, for $n=1$ only $[d y \wedge d z]_{I}$ can be realizable by the germ of a symplectic form. For $n \geq 2$ algebraic restrictions are realizable by (5.1), (5.2) and (5.4). Normal forms in Table 2 are obtained by an obvious change of coordinates. 


\section{REFERENCES}

[A1] V. I. Arnold, First step of local symplectic algebra, Differential topology, infinitedimensional Lie algebras, and applications. D. B. Fuchs' 60th anniversary collection. Providence, RI: American Mathematical Society. Transl., Ser. 2, Am. Math. Soc. 194(44), 1999,1-8.

[AG] V. I. Arnold, A. B. Givental Symplectic geometry, in Dynamical systems, IV, 1-138, Encyclopedia of Matematical Sciences, vol. 4, Springer, Berlin, 2001.

[AVG] V. I. Arnold, S. M. Gusein-Zade, A. N. Varchenko, Singularities of Differentiable Maps, Vol. 1, Birhauser, Boston, 1985.

[D] W. Domitrz, Local symplectic algebra of quasi-homogeneous curves, Fundamentae Mathematicae 204 (2009), 57-86.

[DJZ1] W. Domitrz, S. Janeczko, M. Zhitomirskii, Relative Poincaré lemma, contractibility, quasi-homogeneity and vector fields tangent to a singular variety, Ill. J. Math. 48, No.3 (2004), 803-835.

[DJZ2] W. Domitrz, S. Janeczko, M. Zhitomirskii, Symplectic singularities of varietes: the method of algebraic restrictions, J. reine und angewandte Math. 618 (2008), 197-235.

[DR] W. Domitrz, J. H. Rieger, Volume preserving subgroups of $\mathcal{A}$ and $\mathcal{K}$ and singularities in unimodular geometry, Mathematische Annalen 345 (2009), 783-817.

[DT1] W. Domitrz, Ż. Trȩbska, Symplectic $S_{\mu}$ singularities, Real and Complex Singularities, Contemporary Mathematics, 569, Amer. Math. Soc., Providence, RI, 2012, 45-65.

[DT2] W. Domitrz, Ż. Trȩbska, Symplectic $T_{7}, T_{8}$ singularities and Lagrangian tangency orders, to appear in Proceedings of the Edinburgh Mathematical Society.

[G] M. Giusti, Classification des singularités isolées d'intersections complètes simples, C. R. Acad. Sci., Paris, Sr. A 284 (1977), 167-170 .

[IJ1] G. Ishikawa, S. Janeczko, Symplectic bifurcations of plane curves and isotropic liftings, Q. J. Math. 54, No.1 (2003), 73-102.

[IJ2] G. Ishikawa, S. Janeczko, Symplectic singularities of isotropic mappings, Geometric singularity theory, Banach Center Publications 65 (2004), 85-106.

[K] P. A. Kolgushkin, Classification of simple multigerms of curves in a space endowed with a symplectic structure, St. Petersburg Math. J. 15 (2004), no. 1, 103-126.

[L] E. J. M. Looijenga Isolated Singular Points on Complete Intersections, London Mathematical Society Lecture Note Series 77, Cambridge University Press 1984.

[T] $\quad \dot{\mathrm{Z}}$. Trębska, Symplectic $W_{8}$ and $W_{9}$ singularities, to appear in Journal of Singularities.

[Z] M. Zhitomirskii, Relative Darboux theorem for singular manifolds and local contact algebra, Can. J. Math. 57, No.6 (2005), 1314-1340.

Warsaw University of Technology, Faculty of Mathematics and Information Science, Plac Politechniki 1, 00-661 Warsaw, Poland,

E-mail address: domitrz@mini.pw.edu.pl 